\title{
Successive iteration and positive solutions for a third-order boundary value problem involving integral conditions
}

\author{
Huihui Pang ${ }^{1 *}$, Wenyu Xie ${ }^{1}$ and Limei Cao ${ }^{2}$
}

\section{*Correspondence:}

phh2000@163.com

${ }^{1}$ College of Science, China

Agricultural University, Beijing,

100083, China

Full list of author information is

available at the end of the article

\section{Introduction}

Third-order differential equation arises from many branches of applied mathematics and physics. For example, in the deflection of a curved beam having a constant or varying cross-section, a three-layer beam, electromagnetic waves or gravity driven flows and so on [1].

In the past ten years or so, many authors also studied some third-order differential equation boundary value problems by different types of techniques. For example, in [2], Sun applied the Krasnosel'skii's fixed point theorem to obtain the existence of positive solutions for third-order three-point boundary value problem. In [3], authors studied the uniqueness and existence results for a third-order multi-point boundary value problem by the method of upper and lower solutions.

In [4], Zhou and Ma obtained the existence of positive solutions and established a corresponding iterative scheme for the following third-order boundary value problem:

$$
\left\{\begin{array}{l}
\left(\Phi_{p}\left(u^{\prime \prime}\right)\right)^{\prime}(t)=q(t) f(t, u(t)), \quad 0 \leq t \leq 1, \\
u(0)=\sum_{i=1}^{m} \alpha_{i} u\left(\zeta_{i}\right), \quad u^{\prime}(\eta)=0, \quad u^{\prime \prime}(1)=\sum_{i=1}^{n} \beta_{i} u^{\prime \prime}\left(\theta_{i}\right),
\end{array}\right.
$$

the main tool was the monotone iterative technique.

Boundary value problems with Riemann-Stieltjes integral boundary condition have been considered recently as both multi-point and integral type boundary conditions are treated in a single framework. For more comments on the Riemann-Stieltjes integral boundary condition and its importance, we refer the reader to the papers by Webb and Infante [57] and other related works, such as [8, 9].

(c) 2015 Pang et al. This article is distributed under the terms of the Creative Commons Attribution 4.0 International License (http://creativecommons.org/licenses/by/4.0/), which permits unrestricted use, distribution, and reproduction in any medium, provided you give appropriate credit to the original author(s) and the source, provide a link to the Creative Commons license, and indicate if changes were made. 
In the existing literature, very few papers have dealt with third-order differential equations with Riemann-Stieltjes integral boundary conditions. We found that Graef and Webb [10] studied the following problem:

$$
\left\{\begin{array}{l}
u^{\prime \prime \prime}(t)=g(t) f(t, u(t)), \quad t \in(0,1), \\
u(0)=\alpha[u], \quad u^{\prime}(p)=0, \quad u^{\prime \prime}(1)+\beta[u]=\lambda\left[u^{\prime \prime}\right]
\end{array}\right.
$$

where $p>\frac{1}{2}$, and $\alpha[u], \beta[u]$ and $\lambda[v]$ are linear functionals on $C[0,1]$ given by a RiemannStieltjes integral. The existence of multiple positive solutions is obtained by the application of fixed point index theory. Since $\alpha, \beta$ and $\lambda$ can include both sums and integrals, this boundary condition is more general setup than in (1.1).

In [11], Zhang and Sun investigated the existence of monotone positive solution for the following third-order nonlocal boundary value problem:

$$
\left\{\begin{array}{l}
u^{\prime \prime \prime}(t)+f\left(t, u(t), u^{\prime}(t)\right)=0, \quad 0<t<1, \\
u(0)=0 \\
a u^{\prime}(0)-b u^{\prime \prime}(0)=\alpha[u] \\
c u^{\prime}(1)+d u^{\prime \prime}(1)=\beta[u]
\end{array}\right.
$$

where $\alpha[u]=\int_{0}^{1} u(t) d A(t)$ and $\beta[u]=\int_{0}^{1} u(t) d B(t)$ are linear functionals on $C[0,1]$ given by Riemann-Stieltjes integrals. The main tool is monotone iterative techniques.

Inspired by $[5,10,11]$, in this paper, we apply monotone iterative techniques to obtain the existence and iteration of monotone positive solutions for the following boundary value problem:

$$
\left\{\begin{array}{l}
u^{\prime \prime \prime}(t)=g(t) f\left(t, u(t), u^{\prime}(t), u^{\prime \prime}(t)\right), \quad t \in(0,1), \\
u(0)=\alpha[u], \quad u^{\prime}(p)=0, \quad u^{\prime \prime}(1)+\beta[u]=\lambda\left[u^{\prime \prime}\right]
\end{array}\right.
$$

where $p>\frac{1}{2}, \alpha[u]=\int_{0}^{1} u(s) d A(s), \beta[u]=\int_{0}^{1} u(s) d B(s), \lambda[v]=\int_{0}^{1} v(t) d \Lambda(t)$, and $\alpha[u], \beta[u]$, $\lambda[v]$ are linear functionals on $C[0,1]$ given by the Riemann-Stieltjes integral, $A(t), B(t)$ and $\Lambda(t)$ are suitable functions of bounded variation.

Compared with (1.1)-(1.3), the difficulty of this paper is that nonlinear term $f$ depends on all the lower derivatives of $u$, which leads to complexities to prove the properties of the operator $T$, especially the monotonicity of the operator $T$. In addition, it is worth stating that the first term of our iterative scheme is a simple function or a constant function. Therefore, the iterative scheme is feasible. Under the appropriate assumptions on nonlinear term, this paper is to establish a new and general result on the existence of positive solution to boundary value problem (1.4). An example is also included to illustrate the main results.

\section{Preliminaries}

In this section, we give the definitions and some preliminaries.

Definition 2.1 Let $E$ be a real Banach space. A nonempty closed set $P \subset E$ is said to be a cone provided the following hypotheses are satisfied:

(i) if $u \in P, \lambda \geq 0$, then $\lambda u \in P$;

(ii) if $u \in P$ and $-u \in P$, then $u=0$. 
Definition 2.2 Let the Banach space $E=C^{2}[0,1], u \in E$ is said to be concave on $[0,1]$ if

$$
u\left(\lambda t_{1}+(1-\lambda) t_{2}\right) \geq \lambda u\left(t_{1}\right)+(1-\lambda) u\left(t_{2}\right)
$$

for any $t_{1}, t_{2} \in[0,1]$ and $\lambda \in[0,1]$.

We consider the Banach space $E=C^{2}[0,1]$ equipped with the norm

$$
\|u\|=\max \left\{\max _{0 \leq t \leq 1}|u(t)|, \max _{0 \leq t \leq 1}\left|u^{\prime}(t)\right|, \max _{0 \leq t \leq 1}\left|u^{\prime \prime}(t)\right|\right\} .
$$

Denote

$$
E_{+}=C_{+}^{2}[0,1]=\{u \in E \mid u(t) \geq 0, t \in[0,1]\},
$$

and define the cone $P \subset E$ by

$$
P=\left\{u \in E \mid u(t) \geq 0, u \text { is concave and } u^{\prime}(p)=0\right\} .
$$

Lemma 2.1 For the following boundary value problems,

$$
\gamma^{\prime \prime \prime}(t)=0, \quad \gamma(0)=1, \quad \gamma^{\prime}(p)=0, \quad \gamma^{\prime \prime}(1)=0
$$

and

$$
\delta^{\prime \prime \prime}(t)=0, \quad \delta(0)=0, \quad \delta^{\prime}(p)=0, \quad \delta^{\prime \prime}(1)=1,
$$

we have $\gamma(t) \equiv 1, \delta(t)=p t-t^{2} / 2$, for $t \in[0,1]$.

Lemma $2.2[10]$ Suppose $\lambda[1] \neq 1$. For any $y \in C[0,1]$, the unique solution of the boundary value problem

$$
\left\{\begin{array}{l}
u^{\prime \prime \prime}(t)=y(t), \\
u(0)=0, \quad u^{\prime}(p)=0, \quad u^{\prime \prime}(1)=\lambda\left[u^{\prime \prime}\right]
\end{array}\right.
$$

is given by

$$
u(t)=\left(t p-t^{2} / 2\right) \int_{0}^{1}\left(1+\frac{\Lambda(s)}{1-\lambda[1]}\right) y(s) d s-t \int_{0}^{p}(p-s) y(s) d s+\int_{0}^{t} \frac{(t-s)^{2}}{2} y(s) d s .
$$

Lemma 2.3 Suppose that $1 / 2 \leq p \leq 1, \Lambda(s) \geq 0$ and $\lambda[1]<1$, let $G(t, s)$ be the Green function

$$
G(t, s):=\left(t p-t^{2} / 2\right)\left(1+\frac{\Lambda(s)}{1-\lambda[1]}\right)-t(p-s) H(p-s)+\frac{(t-s)^{2}}{2} H(t-s)
$$

for $0 \leq t \leq 1,0 \leq s \leq 1$, where $H(x-y)=\left\{\begin{array}{ll}0, & x<y, \\ 1, & x \geq y,\end{array}\right.$, we have

$$
0 \leq G(t, s) \leq \Phi(s):= \begin{cases}\frac{p^{2}}{2}+\frac{p^{2}}{2}\left(\frac{\Lambda(s)}{1-\lambda[1]}\right) & \text { if } s \geq p, \\ \frac{s^{2}}{2}+\frac{p^{2}}{2}\left(\frac{\Lambda(s)}{1-\lambda[1]}\right) & \text { if } s<p .\end{cases}
$$


Proof The upper bounds are obtained by finding $\max _{t \in[0,1]} G(t, s)$ for each fixed $s$. Let $Q(s):=1+\frac{\Lambda(s)}{1-\lambda[1]}$. Since $\Lambda(s) \geq 0$, we can get $Q(s) \geq 1$. From (2.5), we have the following formula:

$$
(\partial / \partial t) G(t, s)=(p-t) Q(s)-(p-s) H(p-s)+(t-s) H(t-s) .
$$

We first consider fixed $s \geq p$. The derivative, which is positive for $t<p$, negative for $p<t<s$ and $p \leq s \leq t$. When $t=p$, the derivative becomes zero. Therefore, under our hypothesis, the maximum of $G(t, s)$ for this fixed $s$ occurs when $t=p$. This gives the upper half of the expression for $\Phi$.

When $s<p$, using the fact that $\Lambda(s)>0$, we have, for $t>s$,

$$
\begin{aligned}
G(t, s) & =\left(t p-t^{2} / 2\right)\left(1+\frac{\Lambda(s)}{1-\lambda[1]}\right)-t(p-s)+\frac{(t-s)^{2}}{2} \\
& =\left(t p-t^{2} / 2\right) \frac{\Lambda(s)}{1-\lambda[1]}+\frac{s^{2}}{2} \leq \frac{p^{2}}{2} \frac{\Lambda(s)}{1-\lambda[1]}+\frac{s^{2}}{2} .
\end{aligned}
$$

When $s<p$ and $t \leq s$, we have

$$
\begin{aligned}
G(t, s) & =\left(t p-t^{2} / 2\right)\left(1+\frac{\Lambda(s)}{1-\lambda[1]}\right)-t(p-s) \\
& =\left(t s-t^{2} / 2\right)+\left(t p-t^{2} / 2\right) \frac{\Lambda(s)}{1-\lambda[1]} \leq \frac{s^{2}}{2}+\frac{p^{2}}{2} \frac{\Lambda(s)}{1-\lambda[1]} .
\end{aligned}
$$

Since $Q(s)>1$ and $p-\frac{t}{2} \geq 0$, for $0 \leq t, s \leq 1$, we can easily obtain

$$
0 \leq G(t, s)= \begin{cases}t\left(p-\frac{t}{2}\right) Q(s)+\frac{(t-2)^{2}}{2}, & p \leq s<t \\ t\left(p-\frac{t}{2}\right) Q(s), & p \leq s, t<s \\ t\left(p-\frac{t}{2}\right)\left(\frac{\Lambda(s)}{1-\lambda[1]}\right)+\frac{s^{2}}{2}, & s<p, s<t \\ t\left(p-\frac{t}{2}\right)\left(\frac{\Lambda(s)}{1-\lambda[1]}\right)+t\left(s-\frac{t}{2}\right), & t<s<p .\end{cases}
$$

We always suppose that the following assumptions hold:

$\left(\mathrm{H}_{1}\right) g \in L^{1}[0,1], g \geq 0$, and $\int_{0}^{1} g(s) d s>0$;

$\left(\mathrm{H}_{2}\right) A, B$ are of bounded variation and $g_{A}(s), g_{B}(s) \geq 0$ for a.e. $s$, where

$$
g_{A}(s):=\int_{0}^{1} G(t, s) d A(t) \quad \text { and } \quad g_{B}(s):=\int_{0}^{1} G(t, s) d B(t)
$$

$\left(\mathrm{H}_{3}\right) \gamma \in C[0,1], \gamma(t) \geq 0,0 \leq \alpha[\gamma]<1, \beta[\gamma] \geq 0$;

$\left(\mathrm{H}_{4}\right) \delta \in C[0,1], \delta(t) \geq 0,0 \leq \beta[\delta]<1, \alpha[\delta] \geq 0$;

$\left(\mathrm{H}_{5}\right) \quad D:=(1-\alpha[\gamma])(1-\beta[\delta])-\alpha[\delta] \beta[\gamma]>0$.

Lemma 2.4 [10] For any $y \in C[0,1]$, suppose that $u$ is a solution of the following boundary value problem:

$$
\left\{\begin{array}{l}
u^{\prime \prime \prime}(t)=y(t), \quad t \in(0,1), \\
u(0)=\alpha[u], \quad u^{\prime}(p)=0, \quad u^{\prime \prime}(1)+\beta[u]=\lambda\left[u^{\prime \prime}\right],
\end{array}\right.
$$


then we have

$$
\begin{aligned}
u(t)= & \frac{\gamma(t)}{D}\left[(1-\beta[\delta]) \int_{0}^{1} g_{A}(s) y(s) d s+\alpha[\delta] \int_{0}^{1} g_{B}(s) y(s) d s\right] \\
& +\frac{\delta(t)}{D}\left[\beta[\gamma] \int_{0}^{1} g_{A}(s) y(s) d s+(1-\alpha[\gamma]) \int_{0}^{1} g_{B}(s) y(s) d s\right] \\
& +\int_{0}^{1} G(t, s) y(s) d s .
\end{aligned}
$$

For any $u \in E_{+}, T: P \rightarrow E$ is defined

$$
(T u)(t)=\gamma(t) \alpha\left[g f_{u}\right]+\delta(t) \beta\left[g f_{u}\right]+\int_{0}^{1} G(t, s) g(s) f_{u}(s) d s,
$$

where $f_{u}(s)=f\left(s, u(s), u^{\prime}(s), u^{\prime \prime}(s)\right)$, and

$$
\begin{aligned}
& \alpha\left[g f_{u}\right]=\frac{1}{D}\left[(1-\beta[\delta]) \int_{0}^{1} g_{A}(s) g(s) f_{u}(s) d s+\alpha[\delta] \int_{0}^{1} g_{B}(s) g(s) f_{u}(s) d s\right] \\
& \beta\left[g f_{u}\right]=\frac{1}{D}\left[\beta[\gamma] \int_{0}^{1} g_{A}(s) g(s) f_{u}(s) d s+(1-\alpha[\gamma]) \int_{0}^{1} g_{B}(s) g(s) f_{u}(s) d s\right] .
\end{aligned}
$$

Combining the expression of $\alpha\left[g f_{u}\right], \beta\left[g f_{u}\right]$ with $\left(\mathrm{H}_{1}\right)-\left(\mathrm{H}_{5}\right)$, we can obtain that

$\alpha\left[g f_{u}\right], \beta\left[g f_{u}\right] \geq 0$.

From the definition of $T$, it is obvious that

$$
\begin{aligned}
& (T u)^{\prime \prime}(t)= \begin{cases}-\beta\left[g f_{u}\right]+\int_{0}^{1}(-Q(s)) g(s) f_{u}(s) d s, & t<s, \\
-\beta\left[g f_{u}\right]+\int_{0}^{1}(1-Q(s)) g(s) f_{u}(s) d s, & t>s,\end{cases} \\
& (T u)^{\prime}(t)= \begin{cases}(p-t) \beta\left[g f_{u}\right]+\int_{0}^{1}(p-t) Q(s) g(s) f_{u}(s) d s, & t<p \leq s \text { or } p \leq t \leq s, \\
(p-t) \beta\left[g f_{u}\right]+\int_{0}^{1}[(p-t) Q(s)+(s-p)] g(s) f_{u}(s) d s, & t \leq s<p, \\
(p-t) \beta\left[g f_{u}\right]+\int_{0}^{1}[(p-t) Q(s)+(t-s)] g(s) f_{u}(s) d s, & p \leq s \leq t, \\
(p-t) \beta\left[g f_{u}\right]+\int_{0}^{1}[(p-t) Q(s)+(t-p)] g(s) f_{u}(s) d s, & s \leq t<p \text { or } s<p \leq t .\end{cases}
\end{aligned}
$$

Lemma 2.5 If $\left(\mathrm{H}_{1}\right)-\left(\mathrm{H}_{5}\right)$ are satisfied, $T: P \rightarrow P$ is completely continuous.

Proof Since $(T u)^{\prime \prime}(t) \leq 0, T u$ is concave. From $(T u)^{\prime}(t) \geq 0$ on $[0, p]$ and $(T u)^{\prime}(t) \leq 0$ on $[p, 1]$, we obtain that $(T u)(t)$ is nondecreasing on $[0, p]$ and nonincreasing on $[p, 1]$. Moreover,

$$
(T u)(0)=\gamma(0) \alpha\left[g f_{u}\right]+\delta(0) \beta\left(g f_{u}\right)+\int_{0}^{1} G(0, s) g(s) f_{u}(s) d s \geq 0,
$$

then

$$
(T u)(1)=\gamma(1) \alpha\left[g f_{u}\right]+\delta(1) \beta\left[g f_{u}\right]+\int_{0}^{1} G(1, s) g(s) f_{u}(s) d s \geq 0 .
$$

So, by the concavity of $T$, then $(T u)(t) \geq 0,0 \leq t \leq 1$. Hence, $T: P \rightarrow P$. 
In what follows, we will prove that $T: P \rightarrow P$ is completely continuous. The continuity of $T$ is obvious. Now, we prove that $T$ is compact. Let $\Omega \subset P$ be a bounded set. It is easy to prove that $T(\Omega)$ is bounded and equicontinuous. Then the Arzelà-Ascoli theorem guarantees that $T(\Omega)$ is relatively compact, which means $T$ is compact.

\section{Main results}

For notational convenience, we denote

$$
\begin{aligned}
& L_{A}=\int_{0}^{1} g_{A}(s) g(s) d s, \quad L_{B}=\int_{0}^{1} g_{B}(s) g(s) d s, \quad L_{C}=\int_{0}^{1} Q(s) g(s) d s, \\
& L_{D}=\int_{0}^{1} g(s) d s, \quad L_{E}=\int_{0}^{1} s g(s) d s, \quad L_{F}=\int_{0}^{1}[Q(s)-1] g(s) d s, \\
& L_{G}=\int_{0}^{1} \Phi(s) g(s) d s, \quad L_{H}=\frac{(1-\beta[\delta]) L_{A}+\alpha[\delta] L_{B}}{D}, \\
& L_{I}=\frac{\beta[\gamma] L_{A}+(1-\alpha[\gamma]) L_{B}}{D} .
\end{aligned}
$$

Remark From $\left(\mathrm{H}_{1}\right)-\left(\mathrm{H}_{5}\right)$, we can easily get that the signs of (3.1) and (3.2) are nonnegative. If $g_{A}(s)$ is a piecewise function related to $t$ for $t \in[0,1], L_{A}$ is an expression of $t$. In this case, we denote $L_{A}=\max _{0 \leq t \leq 1}\left\{\int_{0}^{1} g_{A}(s) g(s) d s\right\}$. The same condition is satisfied with $g_{B}(s)$ and $L_{B}$.

We will prove the following existence result.

Theorem 3.1 Assume that $\left(\mathrm{H}_{1}\right)-\left(\mathrm{H}_{5}\right)$ hold, if there are two positive numbers $a_{1}$, a satisfying $a=\max \left\{L_{H}+L_{G}+\frac{p^{2}}{2}\left(L_{I}+L_{C}+L_{D}\right), L_{I}+L_{C}+L_{D}\right\} a_{1}$, and

(S1) $f\left(t, u_{1}, v_{1}, w_{1}\right) \leq f\left(t, u_{2}, v_{2}, w_{2}\right)$ for $0 \leq t \leq 1,0 \leq u_{1} \leq u_{2} \leq a, 0 \leq\left|v_{1}\right| \leq\left|v_{2}\right| \leq a$, $-a \leq w_{2} \leq w_{1} \leq 0$

(S2) $\max _{0 \leq t \leq 1} f(t, a, a,-a) \leq a_{1}$;

(S3) $f(t, 0,0,0) \not \equiv 0$ for $0 \leq t \leq 1$.

Then the boundary value problem (1.4) has positive, nondecreasing on $[0, p]$ nonincreasing on $[p, 1]$ and concave solutions $\omega^{*}, v^{*}$ such that

$$
\begin{aligned}
& 0<\omega^{*} \leq a, \quad 0 \leq\left|\left(\omega^{*}\right)^{\prime}\right| \leq a, \quad-a \leq\left(\omega^{*}\right)^{\prime \prime} \leq 0 \quad \text { and } \\
& \lim _{n \rightarrow \infty} \omega_{n}=\lim _{n \rightarrow \infty} T^{n} \omega_{0}=\omega^{*}, \quad \lim _{n \rightarrow \infty}\left(\omega_{n}\right)^{\prime}=\lim _{n \rightarrow \infty}\left(T^{n} \omega_{0}\right)^{\prime}=\left(\omega^{*}\right)^{\prime}, \\
& \lim _{n \rightarrow \infty}\left(\omega_{n}\right)^{\prime \prime}=\lim _{n \rightarrow \infty}\left(T^{n} \omega_{0}\right)^{\prime \prime}=\left(\omega^{*}\right)^{\prime \prime}
\end{aligned}
$$

where $\omega_{0}(t)=a_{1}\left(L_{H}+L_{G}\right)+a_{1} t\left(p-\frac{t}{2}\right)\left(L_{I}+L_{C}+L_{D}\right)$, and

$$
\begin{aligned}
& 0<v^{*} \leq a, \quad 0 \leq\left|\left(v^{*}\right)^{\prime}\right| \leq a, \quad-a \leq\left(v^{*}\right)^{\prime \prime} \leq 0 \quad \text { and } \\
& \lim _{n \rightarrow \infty} v_{n}=\lim _{n \rightarrow \infty} T^{n} v_{0}=v^{*}, \quad \lim _{n \rightarrow \infty}\left(v_{n}\right)^{\prime}=\lim _{n \rightarrow \infty}\left(T^{n} v_{0}\right)^{\prime}=\left(v^{*}\right)^{\prime}, \\
& \lim _{n \rightarrow \infty}\left(v_{n}\right)^{\prime \prime}=\lim _{n \rightarrow \infty}\left(T^{n} v_{0}\right)^{\prime \prime}=\left(v^{*}\right)^{\prime \prime},
\end{aligned}
$$

where $v_{0}(t)=0$.

Proof We denote

$$
\bar{P}_{a}=\{u \in P \mid\|u\| \leq a\} .
$$


Then, in what follows, we first prove that $T: \bar{P}_{a} \rightarrow \bar{P}_{a}$. If $u \in \bar{P}_{a}$, then $\|u\| \leq a$, we have

$$
\begin{aligned}
& 0 \leq u(t) \leq u(p)=\max _{0 \leq t \leq 1}|u(t)| \leq\|u\| \leq a, \\
& 0 \leq \max _{0 \leq t \leq 1}\left|u^{\prime}(t)\right| \leq\|u\| \leq a, \\
& -a \leq-\|u\| \leq-\max _{0 \leq t \leq 1}\left|u^{\prime \prime}(t)\right| \leq u^{\prime \prime}(t) \leq 0 .
\end{aligned}
$$

So by (S1), (S2) we have

$$
0 \leq f\left(t, u(t), u^{\prime}(t), u^{\prime \prime}(t)\right) \leq \max _{0 \leq t \leq 1} f(t, a, a,-a) \leq a_{1} \quad \text { for } 0 \leq t \leq 1
$$

In fact,

$$
\begin{aligned}
\|T u\| & =\max \left\{\max _{0 \leq t \leq 1}|(T u)(t)|, \max _{0 \leq t \leq 1}\left|(T u)^{\prime}(t)\right|, \max _{0 \leq t \leq 1}\left|(T u)^{\prime \prime}(t)\right|\right\} \\
& =\max \left\{(T u)(p),(T u)^{\prime}(0),-(T u)^{\prime}(1),-(T u)^{\prime \prime}(0)\right\} .
\end{aligned}
$$

By (2.9), (3.1) and (S2), we have

$$
\begin{aligned}
\alpha\left[g f_{u}\right] & =\frac{1}{D}\left[(1-\beta[\delta]) \int_{0}^{1} g_{A}(s) g(s) f_{u}(s) d s+\alpha[\delta] \int_{0}^{1} g_{B}(s) g(s) f_{u}(s) d s\right] \\
& \leq \frac{a_{1}}{D}\left[(1-\beta[\delta]) L_{A}+\alpha[\delta] L_{B}\right]=a_{1} L_{H}, \\
\beta\left[g f_{u}\right] & =\frac{1}{D}\left[\beta[\gamma] \int_{0}^{1} g_{A}(s) g(s) f_{u}(s) d s+(1-\alpha[\gamma]) \int_{0}^{1} g_{B}(s) g(s) f_{u}(s) d s\right] \\
& \leq \frac{a_{1}}{D}\left[\beta[\gamma] L_{A}+(1-\alpha[\gamma]) L_{B}\right]=a_{1} L_{I} .
\end{aligned}
$$

From (3.4), (3.5) and Lemma 2.3, we have

$$
\begin{aligned}
(T u)(p) & =\max _{t \in[0,1]}(T u)(t)=\alpha\left[g f_{u}\right]+\frac{p^{2}}{2} \beta\left[g f_{u}\right]+\int_{0}^{1} G(p, s) g(s) f_{u}(s) d s \\
& \leq a_{1} L_{H}+\frac{p^{2}}{2} a_{1} L_{I}+a_{1} \int_{0}^{1} \Phi(s) g(s) d s \\
& \leq a_{1} L_{H}+\frac{p^{2}}{2} a_{1} L_{I}+a_{1} L_{G} \leq a, \\
-(T u)^{\prime}(1) & = \begin{cases}-\left[(p-1) \beta\left[g f_{u}\right]+(p-1) \int_{0}^{1}(Q(s)-1) g(s) f_{u}(s) d s\right], & p>s, \\
-\left[(p-1) \beta\left[g f_{u}\right]+\int_{0}^{1}[(p-1) Q(s)+1-s] g(s) f_{u}(s) d s\right], & p \leq s\end{cases} \\
& \leq(1-p) a_{1} L_{I}+(1-p) a_{1} L_{C} \leq a_{1}\left(L_{I}+L_{C}\right) \leq a, \\
(T u)^{\prime}(0)= & \begin{cases}p \beta\left[g f_{u}\right]+\int_{0}^{1}[p Q(s)+s-p] g(s) f_{u}(s) d s, & p>s, \\
p \beta\left[g f_{u}\right]+\int_{0}^{1} p Q(s) g(s) f_{u}(s) d s, & p \leq s\end{cases} \\
& \leq p a_{1}\left(L_{I}+L_{C}\right) \leq a, \\
-(T u)^{\prime \prime}(0) & =\beta\left[g f_{u}\right]+\int_{0}^{1} Q(s) g(s) f_{u}(s) d s \leq a_{1}\left(L_{I}+L_{C}\right) \leq a .
\end{aligned}
$$


Thus, we obtain that

$$
\|T u\|=\max \left\{(T u)(p),(T u)^{\prime}(0),-(T u)^{\prime}(1),-(T u)^{\prime \prime}(0)\right\} \leq a .
$$

Hence, we assert that $T: \bar{P}_{a} \rightarrow \bar{P}_{a}$.

Let $\omega_{0}=a_{1} L_{H}+a_{1} L_{G}+a_{1} t\left(p-\frac{t}{2}\right)\left(L_{I}+L_{C}+L_{D}\right)$, for $0 \leq t \leq 1$, then $\omega_{0}(t) \in \bar{P}_{a}$. Let $\omega_{1}=$ $T \omega_{0}, \omega_{2}=T^{2} \omega_{0}$, then $\omega_{1} \in \bar{P}_{a}$ and $\omega_{2} \in \bar{P}_{a}$. We denote $\omega_{n+1}=T \omega_{n}=T^{n} \omega_{0}, n=0,1,2, \ldots$ Since $T: \bar{P}_{a} \rightarrow \bar{P}_{a}$, we have $\omega_{n} \in T \bar{P}_{a} \subseteq \bar{P}_{a}, n=0,1,2, \ldots$ Since $T$ is completely continuous, we assert that $\left\{\omega_{n}\right\}_{n=1}^{\infty}$ is a sequentially compact set. We have

$$
\begin{aligned}
& \omega_{1}(t)=T \omega_{0}(t) \\
& =\alpha\left[g f_{\omega_{0}}\right]+t\left(p-\frac{t}{2}\right) \beta\left[g f_{\omega_{0}}\right]+\int_{0}^{1} G(t, s) g(s) f_{\omega_{0}}(s) d s \\
& \leq a_{1} L_{H}+t\left(p-\frac{t}{2}\right) a_{1} L_{I}+\int_{0}^{1} \Phi(s) g(s) f_{\omega_{0}}(s) d s \\
& \leq a_{1} L_{H}+t\left(p-\frac{t}{2}\right) a_{1} L_{I}+a_{1} L_{G} \\
& \leq a_{1} L_{H}+a_{1} L_{G}+a_{1} t\left(p-\frac{t}{2}\right)\left(L_{I}+L_{C}+L_{D}\right) \\
& =\omega_{0}(t), \quad 0 \leq t \leq 1, \\
& \left|\omega_{1}^{\prime}(t)\right|=\left|\left(T \omega_{0}\right)^{\prime}(t)\right| \\
& \leq \begin{cases}|p-t| \beta\left[g f_{\omega_{0}}\right]+\int_{0}^{1}|p-t| Q(s) g(s) f_{\omega_{0}}(s) d s, & t<p \leq s \text { or } p \leq t<s, \\
|p-t| \beta\left[g f_{\omega_{0}}\right]+\int_{0}^{1}|(p-t) Q(s)-(p-s)| g(s) f_{\omega_{0}}(s) d s, & t<s<p, \\
|p-t| \beta\left[g f_{\omega_{0}}\right]+\int_{0}^{1}|(p-t) Q(s)+(t-s)| g(s) f_{\omega_{0}}(s) d s, & p \leq s \leq t, \\
|p-t| \beta\left[g f_{\omega_{0}}\right]+\int_{0}^{1}|(p-t) Q(s)-(p-t)| g(s) f_{\omega_{0}}(s) d s, & s \leq t<p \text { or } s<p \leq t\end{cases} \\
& \leq \begin{cases}|p-t|\left[a_{1} L_{I}+a_{1} L_{C}\right], & t<s \\
|p-t|\left[a_{1} L_{I}+a_{1} L_{C}+a_{1} L_{D}\right], & t \geq s\end{cases} \\
& \leq a_{1}|p-t|\left(L_{I}+L_{C}+L_{D}\right)=\left|\omega_{0}^{\prime}(t)\right|, \quad 0 \leq t \leq 1, \\
& \omega_{1}^{\prime \prime}(t)=\left(T \omega_{0}\right)^{\prime \prime}(t) \\
& = \begin{cases}-\beta\left[g f_{\omega_{0}}\right]+\int_{0}^{1}(-Q(s)) g(s) f_{\omega_{0}}(s) d s, & t<s, \\
-\beta\left[g f_{\omega_{0}}\right]+\int_{0}^{1}(1-Q(s)) g(s) f_{\omega_{0}}(s) d s, & t \geq s\end{cases} \\
& \geq \begin{cases}-\left[a_{1} L_{I}+a_{1} L_{C}\right], & t<s, \\
-\left[a_{1} L_{I}+a_{1} L_{F}\right], & t \geq s\end{cases} \\
& \geq-a_{1}\left(L_{I}+L_{C}+L_{D}\right)=\omega_{0}^{\prime \prime}(t), \quad 0 \leq t \leq 1 .
\end{aligned}
$$

So

$$
\begin{aligned}
& \omega_{2}(t)=T \omega_{1}(t) \leq T \omega_{0}(t)=\omega_{1}(t), \quad 0 \leq t \leq 1, \\
& \left|\omega_{2}^{\prime}(t)\right|=\left|\left(T \omega_{1}\right)^{\prime}(t)\right| \leq\left|\left(T \omega_{0}\right)^{\prime}(t)\right|=\left|\omega_{1}^{\prime}(t)\right|, \quad 0 \leq t \leq 1, \\
& \omega_{2}^{\prime \prime}(t)=\left(T \omega_{1}\right)^{\prime \prime}(t) \geq\left(T \omega_{0}\right)^{\prime \prime}(t)=\omega_{1}^{\prime \prime}(t), \quad 0 \leq t \leq 1 .
\end{aligned}
$$


Hence, by induction, we have

$$
\omega_{n+1} \leq \omega_{n}, \quad\left|\omega_{n+1}^{\prime}(t)\right| \leq\left|\omega_{n}^{\prime}(t)\right|, \quad \omega_{n+1}^{\prime \prime}(t) \geq \omega_{n}^{\prime \prime}(t), \quad 0 \leq t \leq 1, n=0,1,2, \ldots
$$

Thus, we assert that $\omega_{n} \rightarrow \omega^{*}$, we get $T \omega^{*}=\omega^{*}$ since $T$ is continuous and $\omega_{n+1}=T \omega_{n}$.

Let $v_{0}=0,0 \leq t \leq 1$, then $v_{0}(t) \in \bar{P}_{a}$. Let $v_{1}=T v_{0}, v_{2}=T^{2} v_{0}$, then $v_{1} \in \bar{P}_{a}$ and $v_{2} \in \bar{P}_{a}$. We denote $v_{n+1}=T v_{n}=T^{n} v_{0}, n=0,1,2, \ldots$ Since $T: \bar{P}_{a} \rightarrow \bar{P}_{a}$, we have $v_{n} \in T \bar{P}_{a} \subseteq \bar{P}_{a}$, $n=0,1,2, \ldots$. Since $T$ is completely continuous, we assert that $\left\{v_{n}\right\}_{n=1}^{\infty}$ is a sequentially compact set. We have

$$
\begin{aligned}
& v_{1}(t)=T v_{0}(t)=T v_{0}(t) \geq 0, \quad 0 \leq t \leq 1 \\
& \left|v_{1}^{\prime}(t)\right|=\left|\left(T v_{0}\right)^{\prime}(t)\right|=\left|\left(T v_{0}\right)^{\prime}(t)\right| \geq 0, \quad 0 \leq t \leq 1 \\
& v_{1}^{\prime \prime}(t)=\left(T v_{0}\right)^{\prime \prime}(t)=\left(T v_{0}\right)^{\prime \prime}(t) \leq 0, \quad 0 \leq t \leq 1
\end{aligned}
$$

So

$$
\begin{aligned}
& v_{2}(t)=T v_{1}(t) \geq T v_{0}(t)=v_{1}(t), \quad 0 \leq t \leq 1, \\
& \left|v_{2}^{\prime}(t)\right|=\left|\left(T v_{1}\right)^{\prime}(t)\right| \geq\left|\left(T v_{0}\right)^{\prime}(t)\right|=\left|v_{1}^{\prime}(t)\right|, \quad 0 \leq t \leq 1, \\
& v_{2}^{\prime \prime}(t)=\left(T v_{1}\right)^{\prime \prime}(t) \leq\left(T v_{0}\right)^{\prime \prime}(t)=v_{1}^{\prime \prime}(t), \quad 0 \leq t \leq 1
\end{aligned}
$$

Hence, by induction, we have

$$
v_{n+1} \geq v_{n}, \quad\left|v_{n+1}^{\prime}(t)\right| \geq\left|v_{n}^{\prime}(t)\right|, \quad v_{n+1}^{\prime \prime}(t) \leq v_{n}^{\prime \prime}(t), \quad 0 \leq t \leq 1, n=0,1,2, \ldots
$$

Thus, we assert that $v_{n} \rightarrow v^{*}$, we get $T v^{*}=v^{*}$ since $T$ is continuous and $v_{n+1}=T v_{n}$.

It is well known that the fixed point of the operator $T$ is the solution of boundary value problem (1.4). Therefore, $\omega^{*}$ and $v^{*}$ are positive nondecreasing on $[0, p]$, nonincreasing on $[p, 1]$ and concave solutions of problem (1.4).

Example Let $p=\frac{2}{3}$ and $g(s)=1$, we consider the following boundary value problem:

$$
\left\{\begin{array}{l}
u^{\prime \prime \prime}(t)=\frac{t u}{70}+\frac{1}{540} u^{\prime 2}+e^{-u^{\prime \prime}}, \quad t \in(0,1), \\
u(0)=\alpha[u], \quad u^{\prime}\left(\frac{2}{3}\right)=0, \quad u^{\prime \prime}(1)+\beta[u]=\lambda\left[u^{\prime \prime}\right]
\end{array}\right.
$$

where $\alpha[u]=\int_{0}^{1}(1-s) u(s) d s$ and $\beta[u]=\int_{0}^{1} s u(s) d s$ are nonlocal boundary conditions of integral type. For these boundary conditions, we have $\gamma(t)=1$ and $\delta(t)=\frac{2}{3} t-\frac{t^{2}}{2}$ corresponding to Lemma 2.1. A simple calculation shows that

$$
\begin{aligned}
& \alpha[\gamma]=\frac{1}{2}, \quad \beta[\gamma]=\frac{1}{2}, \quad \alpha[\delta]=\frac{5}{72}, \quad \beta[\delta]=\frac{7}{72}, \\
& D=(1-\alpha[\gamma])(1-\beta[\delta])-\alpha[\delta] \beta[\gamma]=\frac{5}{12}, \\
& L_{A}=\frac{355}{1,296}, \quad L_{B}=\frac{409}{1,296}, \quad L_{C}=\frac{7}{2}, \quad L_{D}=1, \quad L_{E}=\frac{1}{2}, \\
& L_{F}=\frac{5}{2}, \quad L_{G}=\frac{7}{9}, \quad L_{H}=\frac{157}{243}, \quad L_{I}=\frac{191}{270},
\end{aligned}
$$


and $a=\max \left\{L_{H}+L_{G}+\frac{p^{2}}{2}\left(L_{I}+L_{C}+L_{D}\right), L_{I}+L_{C}+L_{D}\right\} a_{1} \approx 5.2 a_{1}$. Then all the hypotheses of Theorem 3.1 are fulfilled with $a=52$ and $a_{1}=10$. It follows from Theorem 3.1 that the boundary value problem (3.6) has two monotone positive solutions $\omega^{*}$ and $v^{*}$ such that

$$
\begin{aligned}
& 0<\omega^{*} \leq 52, \quad 0 \leq\left|\left(\omega^{*}\right)^{\prime}\right| \leq 52, \quad-52 \leq\left(\omega^{*}\right)^{\prime \prime} \leq 0 \quad \text { and } \\
& \lim _{n \rightarrow \infty} \omega_{n}=\lim _{n \rightarrow \infty} T^{n} \omega_{0}=\omega^{*}, \quad \lim _{n \rightarrow \infty}\left(\omega_{n}\right)^{\prime}=\lim _{n \rightarrow \infty}\left(T^{n} \omega_{0}\right)^{\prime}=\left(\omega^{*}\right)^{\prime}, \\
& \lim _{n \rightarrow \infty}\left(\omega_{n}\right)^{\prime \prime}=\lim _{n \rightarrow \infty}\left(T^{n} \omega_{0}\right)^{\prime \prime}=\left(\omega^{*}\right)^{\prime \prime},
\end{aligned}
$$

where $\omega_{0}(t)=-\frac{433}{27} t^{2}+\frac{1,732}{81} t+\frac{3,460}{243}$, and

$$
\begin{aligned}
& 0<v^{*} \leq 66, \quad 0 \leq\left|\left(v^{*}\right)^{\prime}\right| \leq 66, \quad-66 \leq\left(v^{*}\right)^{\prime \prime} \leq 0 \quad \text { and } \\
& \lim _{n \rightarrow \infty} v_{n}=\lim _{n \rightarrow \infty} T^{n} v_{0}=v^{*}, \quad \lim _{n \rightarrow \infty}\left(v_{n}\right)^{\prime}=\lim _{n \rightarrow \infty}\left(T^{n} v_{0}\right)^{\prime}=\left(v^{*}\right)^{\prime}, \\
& \lim _{n \rightarrow \infty}\left(v_{n}\right)^{\prime \prime}=\lim _{n \rightarrow \infty}\left(T^{n} v_{0}\right)^{\prime \prime}=\left(v^{*}\right)^{\prime \prime},
\end{aligned}
$$

where $v_{0}(t)=0$.

\section{Competing interests}

The authors declare that they have no competing interests.

\section{Authors' contributions}

All authors contributed equally to the writing of this paper. All authors read and approved the final manuscript.

\section{Author details}

${ }^{1}$ College of Science, China Agricultural University, Beijing, 100083, China. ${ }^{2}$ School of Mathematics and Physics, University of Science and Technology Beijing, Beijing, 100083, China.

\section{Acknowledgements}

This work was supported by Beijing Higher Education Young Elite Teacher Project (Project No. YETP0322).

Received: 18 May 2015 Accepted: 27 July 2015 Published online: 13 August 2015

\section{References}

1. Gregus, M: Third Order Linear Differential Equations. Mathematics and Its Applications. Reidel, Dordrecht (1987)

2. Sun, Y: Positive solutions of singular third-order three-point boundary value problem. J. Math. Anal. Appl. 306, 589-603 (2005)

3. Lin, $X, \mathrm{Du}, \mathrm{Z}, \mathrm{Liu}, \mathrm{W}$ : Uniqueness and existence results for a third-order nonlinear multi-point boundary value problem. Appl. Math. Comput. 205, 187-196 (2008)

4. Zhou, C, Ma, D: Existence and iteration of positive solutions for a generalized right-focal boundary value problem with p-Laplacian operator. J. Math. Anal. Appl. 324, 409-424 (2006)

5. Webb, JRL, Infante, G: Positive solutions of nonlocal boundary value problems: a unified approach. J. Lond. Math. Soc. 74, 673-693 (2006)

6. Webb, JRL, Infante, G: Nonlocal boundary value problems of arbitrary order. J. Lond. Math. Soc. (2) 79, 238-258 (2009)

7. Webb, JRL: Positive solutions of some higher order nonlocal boundary value problems. Electron. J. Qual. Theory Differ. Equ. 2009, 29 (2009)

8. Graef, JR, Kong, L: Positive solutions for third order semipositone boundary value problems. Appl. Math. Lett. 22, 1154-1160 (2009)

9. Kong, L: Second order singular boundary value problems with integral boundary conditions. Nonlinear Anal. 72 , 2628-2638 (2010)

10. Graef, JR, Webb, JRL: Third order boundary value problems with nonlocal boundary conditions. Nonlinear Anal. 15, 1542-1551 (2009)

11. Zhang, $H$, Sun, J: Existence and iteration of monotone positive solutions for third-order nonlocal BVPs involving integral conditions. Electron. J. Qual. Theory Differ. Equ. 2012, 18 (2012) 